\title{
Phase Equilibria of Quasi-Ternary Systems Consisting of Multicomponent Polymers in a Binary Solvent Mixture II. Role of Initial Concentration and Relative Amount of Polymers Partitioned in Two Phases
}

\author{
Kenji KAMIDE and Shigenobu MATSUDA \\ Textile Research Laboratory, Asahi Chemical Industry Company, Ltd., \\ 11-7 Hacchonawate, Takatsuki, Osaka 569, Japan
}

(Received January 26, 1984)

\begin{abstract}
An attempt was made to clarify by computer experiments the role of initial "concentration" $v_{\mathrm{p}}{ }^{\circ}$ and relative amounts of polymers, partitioned in two phases at the phase equilibrium of quasi-ternary polymer solutions. For this purpose, a polymer with the Schulz-Zimm type molecular weight distribution (the ratio of the weight- to number-average molar volume of the polymer relative to the molar volume of solvents, $X_{w}{ }^{\circ} / X_{n}{ }^{\circ}=2.0$ and $X_{w}=300$ ) was employed under the condition of $v_{\mathrm{p}}{ }^{\mathrm{o}}=1 \times 10^{-5} \sim 0.07$ and the relative amount of the polymer in the polymer-rich phase $\rho_{\mathrm{p}}=1 / 40 \sim 39 / 40$. The phase equilibrium of the quasi-ternary polymer solutions can be approximated with that of the quasi-binary system consisting of multicomponent polymers and a single solvent having the negative concentration dependence of the polymer-solvent interaction parameter $\chi$. As $\rho_{\mathrm{p}}$ increases, both the phase volume ratio $R$ and the $X_{w} / X_{n}$ of the polymer in the polymer-rich phase decrease and then increase after passing through a minimum.

KEY WORDS Quasi-Ternary Polymer Solution / Phase Equilibrium / Initial Concentration / Relative Amount of Polymer /
\end{abstract}

In a previous paper ${ }^{1}$ we established a theory and methodology for computer experiments for the two-phase equilibrium of a quasiternary system consisting of a multicomponent polymer, solvent 1 , and solvent 2 at constant temperature and pressure. We investigated in detail the effects of the solution powers of two solvents on the phase separation characteristics of solutions of a polymer having a SchulzZimm molecular weight distribution. The molar volumes of these two solvents were assumed to be identical, and the weight-average molar volume of the polymer relative to that of the solvents, $X_{w}{ }^{\circ}$, to be 300 (here, the suffix o denotes the original polymer) when the polymer volume fraction of the starting solution, $v_{\mathrm{p}}^{\mathrm{s}}=0.01$ and the relative amount of the polymer partitioned in the polymerrich phase, $\rho_{\mathrm{p}}=1 / 15$.

In this paper, we study the role of phase separation conditions such as the initial polymer volume fraction $v_{\mathrm{p}}{ }^{\mathrm{o}}$ and $\rho_{\mathrm{p}}$ and the relative amount of the polymer partitioned in the polymer-lean phase $\rho_{\mathrm{s}}\left(\equiv 1-\rho_{\mathrm{p}}\right)$, in the phase equilibrium of quasi-ternary polymer solutions. These points have already been studied by Kamide et $a l^{2-12}$ in a systematic manner for quasi-binary systems consisting of a multicomponent polymer and single solvent.

\section{Computer Experiment}

The simulation procedure, established in the previous paper, ${ }^{1}$ was employed with hardly any modification. A polymer having a SchulzZimm type molecular weight distribution and $X_{w}{ }^{\circ}=300$ and $X_{n}{ }^{\circ}$ (number average) $=150$ was used as the starting original polymer in the computer experiments. The hypothetical phase equilibrium calculation was carried out with the electric computer, IBM 4341 II. The total 
number of components comprising the original polymer was 1500 .

First, one gram of the original polymer (density, $1 \mathrm{~g} \mathrm{~cm}^{-3}$; in this case the volume of the polymer $V_{3}{ }^{\circ}=1 \mathrm{ml}$ ) was dissolved in $V_{1}{ }^{\circ} \mathrm{ml}$ of solvent 1 to give a solution of the polymer volume fraction $v_{\mathrm{p}}^{\mathrm{s}}\left(\equiv 1 /\left(1+V_{1}^{\mathrm{o}}\right)\right)$, to which $V_{2} \mathrm{ml}$ of solvent 2 were added. The solution thus prepared had a total volume $V$ $\left(=V_{3}^{\circ}+V_{1}^{\circ}+V_{2}\right)$, and $v_{\mathrm{p}}^{\circ}$ given by

$$
v_{\mathrm{p}}{ }^{\mathrm{o}}=V_{3}{ }^{\mathrm{o}} /\left(V_{3}{ }^{\mathrm{o}}+V_{1}{ }^{\mathrm{o}}+V_{2}\right)=1 /\left(1+V_{1}{ }^{\mathrm{o}}+V_{2}\right)
$$

and was allowed to attain phase equilibrium. $V_{2}$ is related to the phase volume ratio $R$, the volume fraction of solvent 2 in the polymerlean, and -rich phases, $v_{2(1)}$ and $v_{2(2)}$, respectively, and the total volume of solution $V$ by the relation,

$$
V_{2}=\frac{V}{R+1}\left(R v_{2(1)}+v_{2(2)}\right)
$$

Using eq 2 we can express the total volume $V$ in the form

$$
V=\frac{(R+1)\left(V_{3}{ }^{\circ}+V_{1}{ }^{\circ}\right)}{R+1-R v_{2(1)}-v_{2(2)}}
$$

Combination of eq 1 and 3 gives

$$
\begin{aligned}
v_{\mathrm{p}}^{\mathrm{o}} & =\frac{V_{3}^{\mathrm{o}}\left(R+1-R v_{2(1)}-v_{2(2)}\right)}{(R+1)\left(V_{3}^{\mathrm{o}}+V_{1}^{\mathrm{o}}\right)} \\
& =\frac{R+1-R v_{2(1)}-v_{2(2)}}{(R+1)\left(1+V_{1}^{\mathrm{o}}\right)}
\end{aligned}
$$

As shown in the previous paper, ${ }^{1} v_{\mathrm{p}}{ }^{\mathrm{o}}$ can be considered constant by using an adequate $V_{1}{ }^{\circ}$ value in eq 4 as a precondition, if necessary. The computer experiment on a quasi-binary system consisting of a multicomponent polymer in a single solvent was performed by Kamide et al. and polymer molecular weight distribution was the same as that employed in the present calculation of a quasi-ternary system and the concentration dependence of $\chi$ parameter defined by eq 5 was taken into consideration

$$
\chi=\chi_{\mathrm{o}}\left(1+p v_{\mathrm{p}}\right)
$$

where $\chi_{o}$ is a constant independent of $v_{p}$.

\section{RESULTS AND DISCUSSION}

For any quasi-ternary system consisting of a multicomponent polymer, solvent 1 and solvent 2 , three thermodynamic interaction parameters between solvent 1 , solvent 2 and the polymer $3, \chi_{12}, \chi_{13}$, and $\chi_{23}$, can be clearly determined at constant temperature and pressure. The phase equilibrium may or may not occur under the given preconditions of $\rho_{\mathrm{p}}$ and $v_{\mathrm{p}}{ }^{\mathrm{o}}$. Note that the volume ratio of solvent 1 to solvent 2, at phase equilibrium, $V_{1} / V_{2}$ is not always constant when $v_{\mathrm{p}}{ }^{\circ}$ is changed while keeping $\rho_{\mathrm{p}}$ constant. This is exemplified in Figure 1 . With diminishing $v_{\mathrm{p}}{ }^{\mathrm{o}}$, the content of solvent 1 decreases, indicating the composition of a binary solvent mixture to change depending on the operating conditions and, in this regard, the quasi-ternary system to be much more complicated than a quasi-binary system.

Figure 2 shows the change in the total composition (i.e., the composition of the sum of the polymer-lean and -rich phases and that immediately before the phase separation) with $v_{\mathrm{p}}^{\mathrm{s}}$. Here $v_{1}$ and $v_{2}$ are the volume fractions of solvent 1 and solvent 2 in a quasi-ternary system as defined by $v_{1}=V_{1}{ }^{\circ} /\left(V_{1}{ }^{\circ}+V_{2}+V_{3}{ }^{\circ}\right)$ and $v_{2}=V_{2} /\left(V_{1}{ }^{\circ}+V_{2}+V_{3}^{\circ}\right)$. The phase equilibrium conditions are the same as those in Figure 1. A rapid increase in $v_{2}$ with a increase in $\rho_{\mathrm{p}}$ is observed at constant $v_{\mathrm{p}} \mathrm{s}$.

Figure 3 shows the phase diagrams obtained for solutions with $v_{\mathrm{p}}^{\mathrm{s}}=0.001 \sim 0.07$. In this figure coexistence curves and tie-lines for $\rho_{\mathrm{p}}=$ $1 / 15 \sim 14 / 15$ are shown. The same set of $\chi_{12}$, $\chi_{13}$, and $\chi_{23}$ as those in Figures 1 and 2 are employed also. As $v_{\mathrm{p}}{ }^{\mathrm{s}}$ decreases, the coexistence curves slightly shift to the $v_{2}$ apex. A significant variation in coexistence curves with $v_{\mathrm{p}}^{\mathrm{s}}$ (or $v_{\mathrm{p}}{ }^{\mathrm{o}}$ ) sharply reflects the polymolecularity of the original polymer sample. Note that the coexistence curve of the strictly mo- 


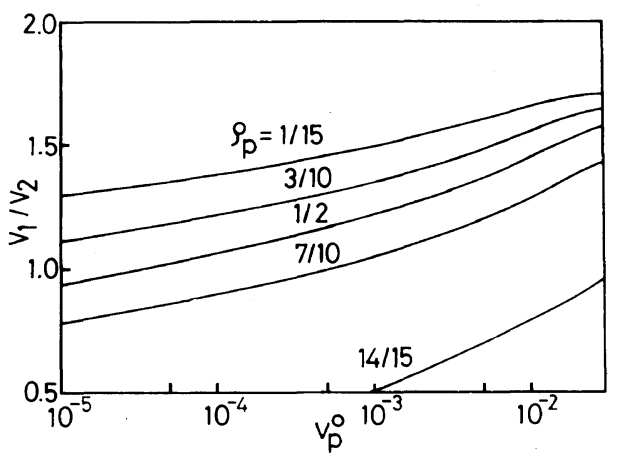

Figure 1. Change in volume ratio, $V_{1} / V_{2}$, of solvent 1 to solvent 2 with the initial volume fraction $v_{\mathrm{p}}{ }^{\circ}$ of the solution in which the two phase separation occurs. The original polymer, Schulz-Zimm distr., $X_{w}{ }^{\circ}=300$, $X_{w}{ }^{\circ} / X_{n}{ }^{\circ}=2.0 ; \chi_{12}=0.48, \chi_{13}=0.20, \chi_{23}=1.20$.

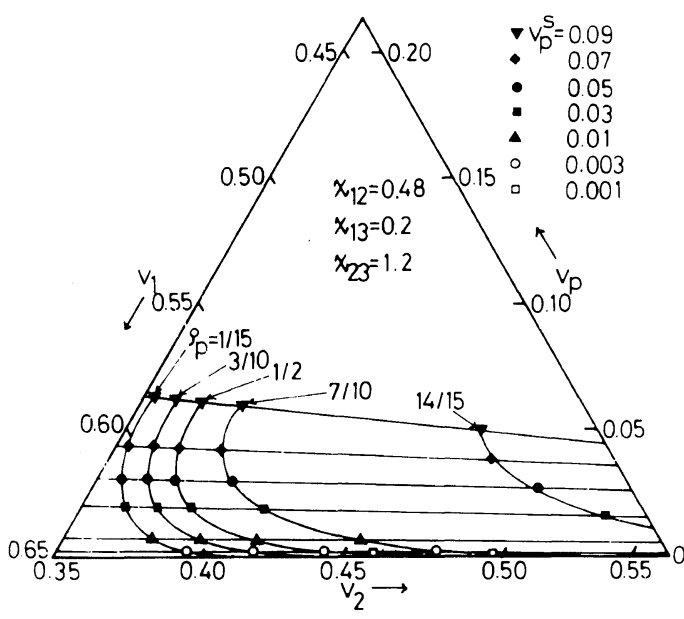

Figure 2. Change in total composition of the solution in which the phase separation occurs, with the starting polymer volume fraction $v_{\mathrm{p}}^{\mathrm{s}}$. The original polymer, Schulz-Zimm distr., $X_{w}{ }^{\circ}=300, X_{w}{ }^{\circ} / X_{n}{ }^{\circ}=2.0 ; \chi_{12}=0.48$, $\chi_{13}=0.20, \chi_{23}=1.20 ; v_{\mathrm{p}}^{\mathrm{s}}, 0.09(\boldsymbol{\nabla}), 0.07(\diamond), 0.05(\boldsymbol{\bullet})$, $0.03(\square), 0.01(\mathbf{\Delta}), 0.003(\bigcirc), 0.001(\square)$.

nodisperse polymer solution is, of course, absolutely independent of $v_{\mathrm{p}}{ }^{\mathrm{o}}$ and $v_{\mathrm{p}}{ }^{\mathrm{s}}$. The coexistence curve at the polymer rich phase side is almost parallel to the $v_{1}$ axis, irrespective of $v_{\mathrm{p}}^{\mathrm{s}}$, suggesting that the volume fraction of solvent 2 in the polymer-rich phase $v_{2(2)}$ is nearly constant, regardless of $\rho_{\mathrm{p}}$.

In two coexisting phases, $v_{2}$ can be regarded

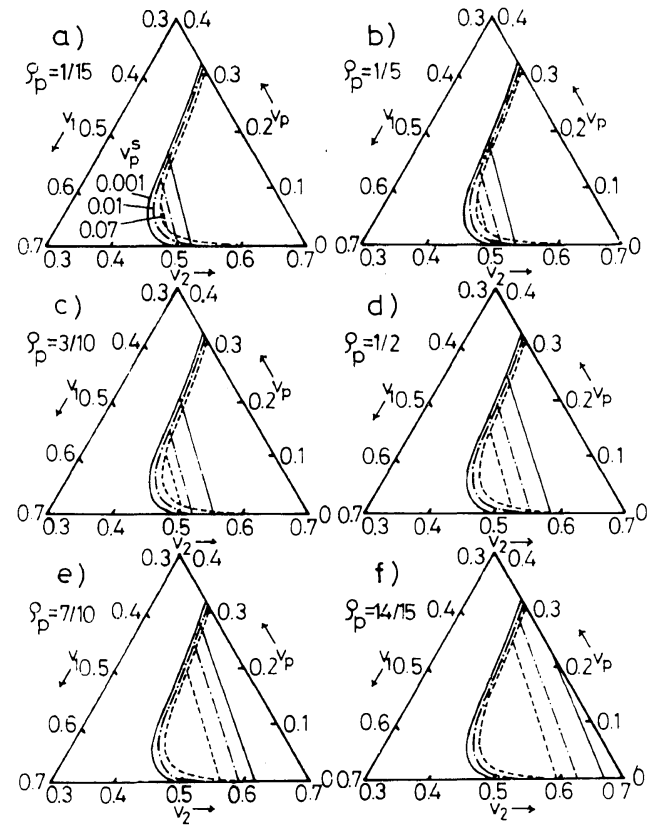

Figure 3. Effects of the starting polymer volume fraction $v_{\mathrm{p}}^{\mathrm{s}}$ and the relative amount of the polymer in a polymer-rich phase $\rho_{\mathrm{p}}$ on the phase diagram of quasiternary polymer solutions. The original polymer, Schulz-Zimm distr., $X_{w}{ }^{\circ}=300, X_{w}{ }^{\circ} / X_{n}{ }^{\circ}=2.0 ; \chi_{12}=0.48$, $\chi_{13}=0.20, \chi_{23}=1.00 ; v_{\mathrm{p}}^{\mathrm{s}}=0.07$ (broken line), 0.01 (chain line), 0.001 (full line); a) $\rho_{\mathrm{p}}=1 / 15$, b) $\rho_{\mathrm{p}}=1 / 5$, c) $\rho_{\mathrm{p}}=$ $3 / 10$, d) $\rho_{\mathrm{p}}=1 / 2$, e) $\rho_{\mathrm{p}}=7 / 10$, f) $\rho_{\mathrm{p}}=14 / 15$.

as roughly the same, but $v_{1}$ and $v_{\mathrm{p}}$ in the polymer-rich phase differ remarkably from those in the polymer-lean phase. That is, in the polymer-rich phase $v_{\mathrm{p}}$ is larger and $v_{1}$, smaller than in the polymer lean phase.

As $v_{\mathrm{p}}{ }^{\mathrm{s}}$ decreases, a tie-line for a given $\rho_{\mathrm{p}}$ shifts to the $v_{2}-v_{\mathrm{p}}$ axis. Of course, this effect of $v_{\mathrm{p}}^{\mathrm{s}}$ on the tie line can be explained reasonably by the polymolecularity of the original polymer sample. At $v_{\mathrm{p}}{ }^{\mathrm{s}}=0.001$, the polymer volume fraction in the polymer-lean phase, $v_{p(1)}$, can be approximated as zero, but this approximation can never be approved for higher $v_{\mathrm{p}}^{\mathrm{s}}$ solutions. Even for a solution with $v_{\mathrm{p}}^{\mathrm{s}}=0.001$, we cannot calculate, with good accuracy, other physical quantities characterizing the phase equilibrium assuming $v_{\mathrm{p}(1)}=0$.

The above-mentioned characteristics of a 


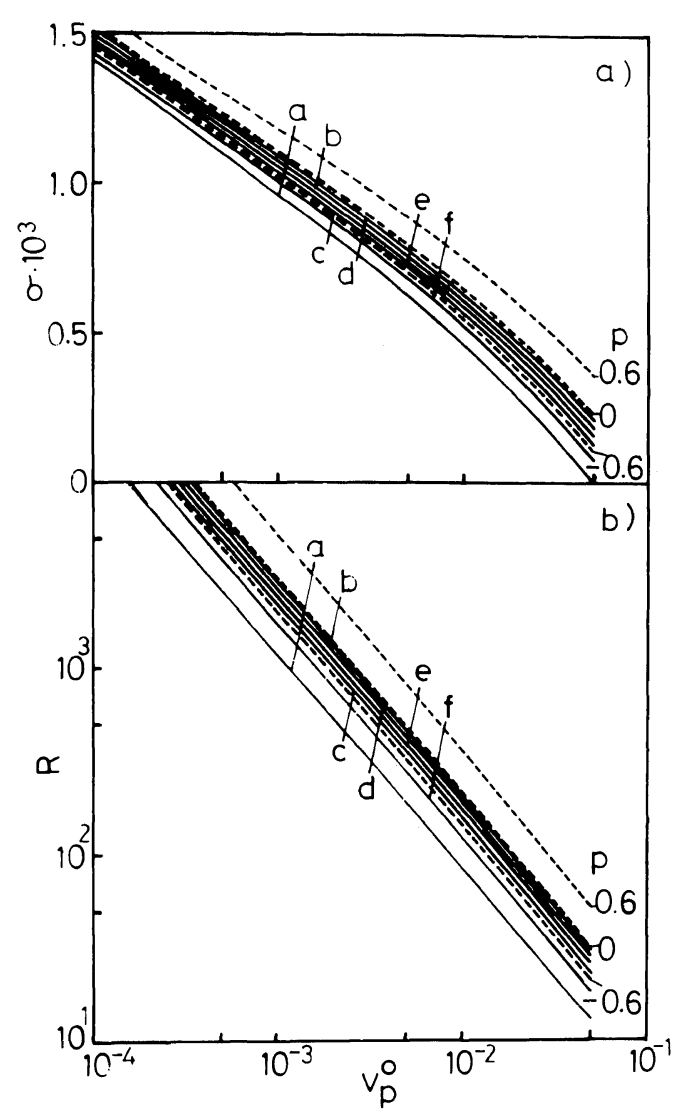

Figure 4. Effects of the $\chi_{12}, \chi_{13}$, and $\chi_{23}$ parameters on the $\sigma$ or $\mathrm{R}$ vs the initial polymer volume fraction $v_{\mathrm{p}}{ }^{\circ}$ relations. The original polymer, Schulz-Zimm distr., $X_{w}{ }^{\circ}=300, X_{w}{ }^{\circ} / X_{n}{ }^{\circ}=2.0 ; \rho_{\mathrm{p}}=1 / 15 ; \mathrm{a}, \chi_{12}=1.30, \chi_{13}=$ $0.20, \chi_{23}=1.0 ; \mathrm{b}, \chi_{12}=0.05, \chi_{13}=0.20, \chi_{23}=1.0 ; \mathrm{c}, \chi_{12}=$ $0.48, \chi_{13}=0.05, \chi_{23}=1.0 ; \mathrm{d}, \chi_{12}=0.48, \chi_{13}=0.50, \chi_{23}=$ 1.0 ; e, $\chi_{12}=0.48, \chi_{13}=0.20, \chi_{23}=1.0$; f, $\chi_{12}=0.48, \chi_{13}=$ $0.20, \chi_{23}=1.4$.

phase diagram of the quasi-ternary system were qualitatively observed by actual experiments on polyethyrene/benzylether/ethanol, ${ }^{13}$ polystyrene/benzyl-benzoate/acetonitrile ${ }^{13}$ and polystyrene/benzene/methanol. ${ }^{14}$

Figure 4 shows the relations among the partition coefficient $\sigma$, phase volume ratio $R$ and $v_{\mathrm{p}}{ }^{\circ}$. The full line in the figure corresponds to a given combination of three $\chi$ parameters. Figure 4 also shows the data (as broken line) for quasi-binary solutions, in which the molecular characteristics of the original polymer
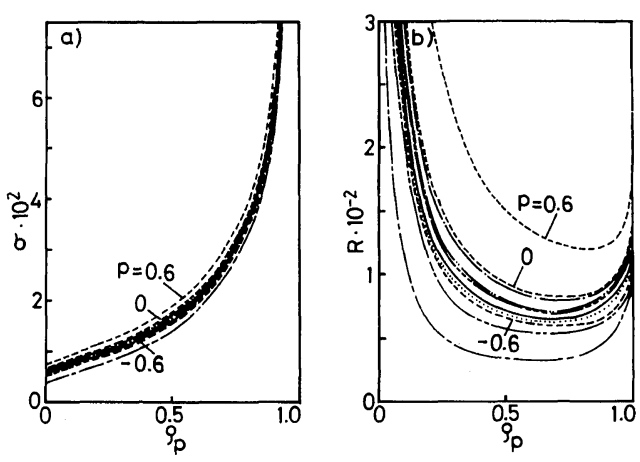

Figure 5. Effects of the $\chi_{12}, \chi_{13}$, and $\chi_{23}$ parameters of the relations between the partition coefficient $\sigma$ or the phase volume ratio $R$ and the relative amount of polymer partitioned in a polymer-rich phase $\rho_{\mathrm{p}}$. The original polymer, Schulz-Zimm distr., $X_{w}{ }^{0}=300, X_{w}{ }^{\circ} / X_{n}{ }^{\circ}=$ $2.0 ; v_{\mathrm{p}}{ }^{\circ}=0.005 ;-, \chi_{12}=0.48, \chi_{13}=0.20, \chi_{23}=1.0$; $-\cdots-, \chi_{12}=0, \chi_{13}=0.2, \chi_{23}=1.0 ;-\cdots-, \chi_{12}=0.9$, $\chi_{13}=0.2, \chi_{23}=1.0 ;-\cdots, \chi_{12}=1.4, \chi_{13}=0.2, \chi_{23}=1.0$; $-\cdot-, \chi_{12}=0.48, \chi_{13}=0.5, \chi_{23}=1.0 ;-\cdots-, \chi_{12}=0.48$, $\chi_{13}=0.2, \chi_{23}=0.8 ; \cdots \cdots \cdots, \chi_{12}=0.48, \chi_{13}=0.2, \chi_{23}=1.3$.

are the same as those used for quasi-ternary solutions and the number on each line is a $p$ parameter. The relations between $\chi_{0}$ and $v_{p}{ }^{\circ}$ for quasi-binary solutions have already been discussed in detail by Kamide and Sugamiya. ${ }^{15}$ The $\sigma$ as well as $R$ decreases with an increase in $v_{\mathrm{p}}{ }^{\mathrm{o}}$. As $\chi_{12}$ and $\chi_{23}$ become smaller and $\chi_{13}$ larger, both $\sigma$ and $R$ increase. For any combination of $\chi_{12}, \chi_{13}$, and $\chi_{23}$, the same $\sigma v s . v_{\mathrm{p}}{ }^{\circ}$ or $R v s . v_{\mathrm{p}}{ }^{\mathrm{o}}$ relations as those for quasi-binary solutions assuming $p=0$ can be obtained. That is, for a given $\rho_{\mathrm{p}}$ and a given $v_{\mathrm{p}}{ }^{\circ}$, the quasiternary system always affords smaller $\sigma$ and $R$ than does the quasi-binary system. The lines for the quasi-binary system with $p=0$ can be regarded as asymptotic lines attainable by the quasi-ternary system. The higher fractionation efficiency for a quasi-binary system was experimentally demonstrated by Kamide et al. ${ }^{16}$ using polystyrene.

Figure 5 shows the effect of $\rho_{\mathrm{p}}$ on $\sigma$ and $R$ for the quasi-ternary (full line) and -binary (broken line) solutions of $v_{\mathrm{p}}{ }^{\mathrm{o}}=0.005$ of the polymer having the Schulz-Zimm distribution $\left(X_{w}{ }^{\circ}=300, X_{n}{ }^{o}=150\right)$. As $\rho_{\mathrm{p}}$ increases from zero to unity, $\sigma$ increases monotonously, but $R$ 
decreases first and increases after passing through a minimum $\left(\rho_{\min }\right)$. As described before, $\sigma$ and $R$, both calculated for the quasiternary solution, do not exceed, by any combination of the three $\chi$ parameters, those for the quasi-binary solution with $\mathrm{p}=0$. A decrease in $\chi_{12}$ and $\chi_{23}$ and an increase in $\chi_{13}$ bring about an increase in $\sigma$ and $R$.

Figure 6 shows the effect of $v_{\mathrm{p}}{ }^{0}$ on the relations between $\sigma$ (or $R$ ) and $\rho_{\mathrm{p}}$. In this case, $\chi_{12}=0.48, \chi_{13}=0.2$, and $\chi_{23}=1.3$ were assumed. The characteristic feature of $\sigma$ (or $R$ ) $v s . \rho_{\mathrm{p}}$ relations in Figure 5 holds its validity over a wide range of $v_{\mathrm{p}}{ }^{\circ}$, from $1 \times 10^{-5}$ to $1 \times 10^{-2}$. That is, as $\rho_{\mathrm{p}}$ increases, $\sigma$ as well as $\mathrm{d} \sigma / \mathrm{d} \rho_{\mathrm{p}}$ increases monotonously, but $R$ decreases first and increases after passing through minimum $\rho_{\mathrm{p}, \min }$ at any $v_{\mathrm{p}}{ }^{\mathrm{o}}$ investigated. $\rho_{\mathrm{p}, \min }$ is maintained almost constant, irrespective of $v_{\mathrm{p}}{ }^{\circ}$.

Figure 7 shows the relationship between $\rho_{\mathrm{p}}$ and $v_{\mathrm{p}}{ }^{\circ}$, which yield the same value of $\sigma$ or $R$. In a smaller $v_{\mathrm{p}}{ }^{\mathrm{o}}$ region, $\sigma$ is evidently $\rho_{\mathrm{p}}$-dependent and in the larger $v_{\mathrm{p}}{ }^{\circ}$ region (particularly at smaller $\left.\rho_{\mathrm{p}}\right) \sigma$ is dependent of $v_{\mathrm{p}}{ }^{\circ}$ and of $\rho_{\mathrm{p}}$. Over an extremely wide $v_{\mathrm{p}}{ }^{0}$ range, $R$ is strongly $v_{\mathrm{p}}{ }^{\circ}$-dependent and the feature of its
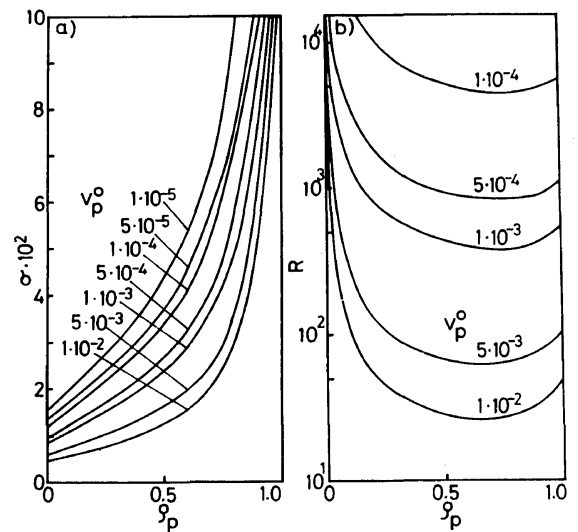

Figure 6. Effect of the initial polymer volume fraction $v_{\mathrm{p}}{ }^{\circ}$ on the relations between the partition coefficient $\sigma$ or the phase volume ratio $R$ and the relative amount of the polymer in a polymer-rich phase $\rho_{\mathrm{p}}$. The original polymer, Schulz-Zimm distr., $X_{w}{ }^{\circ}=300, X_{w}{ }^{\circ} X_{n}{ }^{\circ}=2.0$; $\chi_{12}=0.48, \chi_{13}=0.20, \chi_{23}=1.30$. dependence is almost the same. The dependence of $\rho_{\mathrm{p}}$ on $v_{\mathrm{p}}{ }^{\mathrm{o}}$ at a given $R,\left(\mathrm{~d} \rho_{\mathrm{p}} / \mathrm{d} v_{\mathrm{p}}{ }^{\circ}\right)_{\mathrm{R}}$ changes its sign from positive to negative as $\rho_{\mathrm{p}}$ decreases.

For any value of $\rho_{\mathrm{p}}$, both $\sigma$ and $R$ increases with $v_{\mathrm{p}}{ }^{\mathrm{o}}$ and for any value of $v_{\mathrm{p}}{ }^{\mathrm{o}}, \sigma$ increases with $\rho_{\mathrm{p}}$, but $R$ is minimum at $\rho_{\mathrm{p}} \cong 0.7$. This confirms the general characteristics in Figure 6. Figure 8 shows the relationships among $v_{\mathrm{p}}{ }^{\circ}$, $\rho_{\mathrm{p}}, \sigma$ and $R$ obtained at phase equilibrium. As Kamide and his coworkers pointed out for a quasi-binary system, ${ }^{10}$ under given external

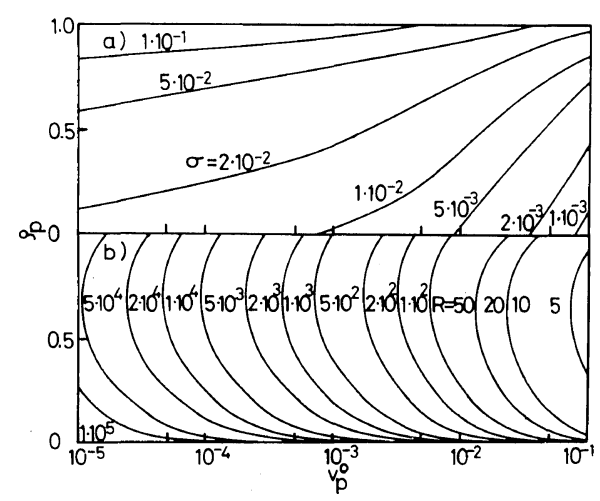

Figure 7. Relationships among $\rho_{\mathrm{p}}, v_{\mathrm{p}}^{\mathrm{o}}$, and $\sigma$ (upper part) and among $\rho_{\mathrm{p}}, v_{\mathrm{p}}{ }^{\circ}$, and $R$ (lower part) for a quasiternary polymer solutions. The original polymer, Schulz-Zimm distr., $X_{w}{ }^{\circ}=300, X_{w}{ }^{\circ} / X_{n}{ }^{\circ}=2.0 ; \chi_{12}=0.48$, $\chi_{13}=0.20, \chi_{23}=1.30$.

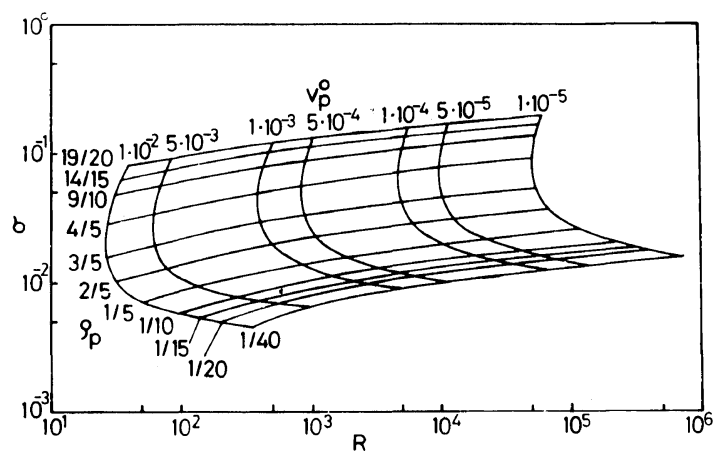

Figure 8. Relations among the initial polymer fraction $v_{\mathrm{p}}^{\circ}$, the relative amount of the polymer in a polymer-rich phase $\rho_{\mathrm{p}}$, the partition coefficient $\sigma$, and the phase volume ratio $R$ for quasi-ternary polymer solution. The original polymer, Schulz-Zimm distr., $X_{w}{ }^{\circ}=300$, $X_{w}{ }^{\circ} / X_{n}{ }^{\circ}=2.0 ; \chi_{12}=0.48, \chi_{13}=0.20, \chi_{23}=1.30$. 


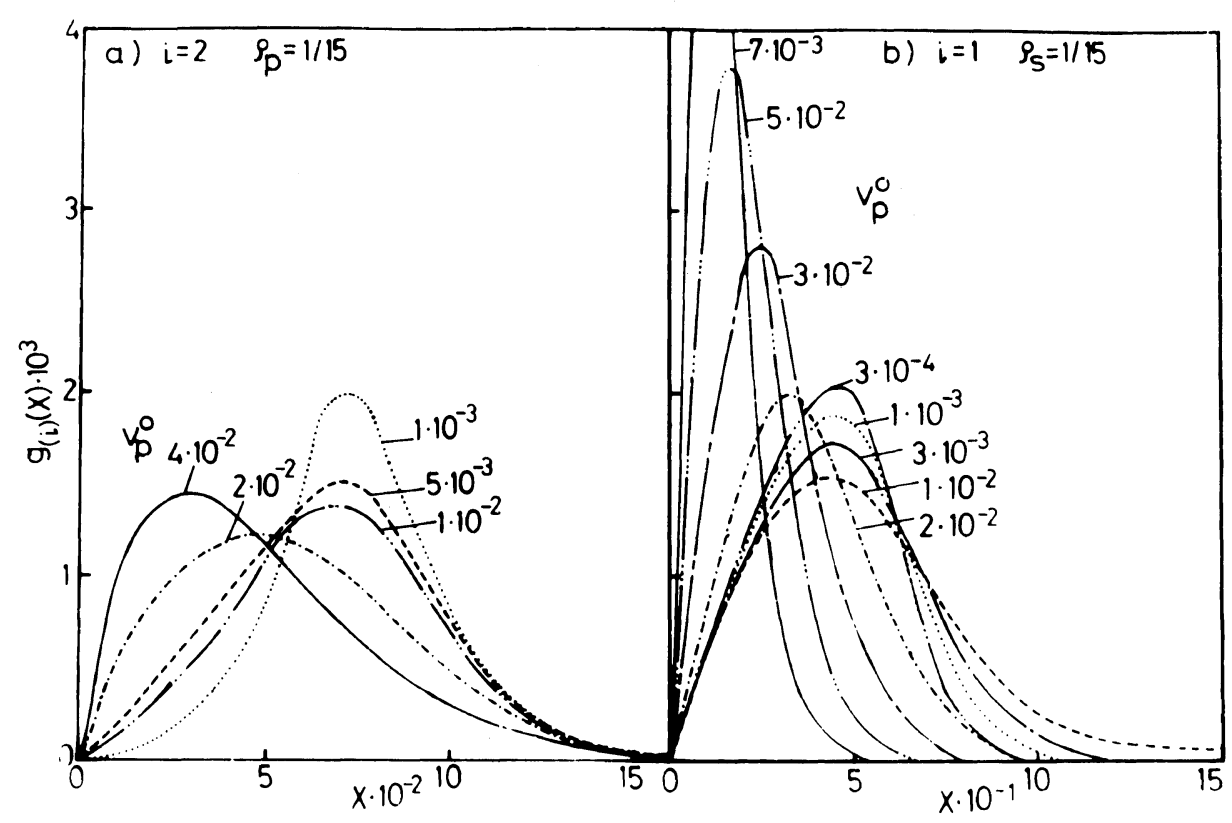

Figure 9. Differential MWD curve $g_{(i)}(X)$ of the polymers in a polymer-rich phase $(i=2)$ or in a polymerlean phase $(i=1)$, separated at $\rho_{\mathrm{p}}=1 / 15$ (a) or 14/15 (i.e., $\rho_{\mathrm{s}}=1 / 15$ ) (b) from different $v_{\mathrm{p}}{ }^{\circ}$ quasiternary solutions (numbers at curves). The original polymer, Schulz-Zimm distr., $X_{w}{ }^{\circ}=300, X_{w}{ }^{\circ} / X_{n}{ }^{\circ}=$ $2.0 ; \chi_{12}=0.48, \chi_{13}=0.20, \chi_{23}=1.30$.

operating conditions $\left(v_{\mathrm{p}}^{\mathrm{o}}\right.$ and $\left.\rho_{\mathrm{p}}\right), \sigma$ together with $R$ are unambiguously determined. In other words, a given pair of $v_{\mathrm{p}}{ }^{\circ}$ and $\rho_{\mathrm{p}}$ corresponds rigorously to a specialized pair of $\sigma$ and $R$ as independent separation variables even for a quasi-ternary system, provided the molecular characteristics of the original polymer and three $\chi$ parameters are given in advance.

It should be remarked that $\sigma$ and $R$ may vary within a network structure in Figure 8 corresponding to a change in $v_{\mathrm{p}}{ }^{\circ}$ ranging from $1 \times 10^{-5}$ to $1 \times 10^{-2}$ and to a change in $\rho_{\mathrm{p}}$ from $1 / 40$ to $19 / 20$. As $v_{\mathrm{p}}{ }^{\mathrm{o}}$ reduces with keeping $\rho_{\mathrm{p}}$ constant, both $\sigma$ and $R$ (particularly, $R$ ) increase progressively and with increasing $\rho_{\mathrm{p}}$ at a given $v_{\mathrm{p}}^{\mathrm{o}}, \sigma$ increase monotonously and $R$ decreases in a small $\rho_{\mathrm{p}}$ region, then increases slightly after passing through the minimum.

Figure 9a exemplifies the normalized molecular weight distribution (MWD) curves $g_{(2)}(X)$ in some polymers remained in the polymer-rich phase, which is phase-separated, keeping $\rho_{\mathbf{p}}=1 / 15$, from the solutions of various concentration $v_{\mathrm{p}}{ }^{\mathrm{o}} \cdot g_{(2)}(X)$ for the polymers isolated from a comparatively concentrated solution shows a peak at a lower $X$ (and this $X$ is designed as $X_{\mathrm{p}}$ ), and approximated as $X_{\mathrm{p}}$ for the original polymer. As $v_{\mathrm{p}}{ }^{\circ}$ decrease, the peak of the MWD curve moves from a lower to higher $X$ region, without changing practically the upper and lower limits of MWD and this shift results in a sharpening of a MWD.

Figure $9 \mathrm{~b}$ shows a normalized MWD curve $g_{(1)}(X)$ of some polymers partitioned in the polymer-lean phase, which is phase-separated, keeping $\rho_{\mathrm{s}}=1 / 15$ (i.e., $\rho_{\mathrm{s}}=14 / 15$ ), from the solutions of various $v_{\mathrm{p}}{ }^{\circ}$. The higher $X$ region in MWD is notably changed by $v_{\mathrm{p}}{ }^{\circ}$ and as $v_{\mathrm{p}}{ }^{\circ}$ is lowered, $X_{\mathrm{p}}$ of $g_{(1)}(X)$ decreases with a remarkable peak sharpening. The shift of $X_{\mathrm{p}}$ and peak sharpening contribute in reverse manner to MWD and in this case, the effect of the latter is significantly larger on MWD than that of the 


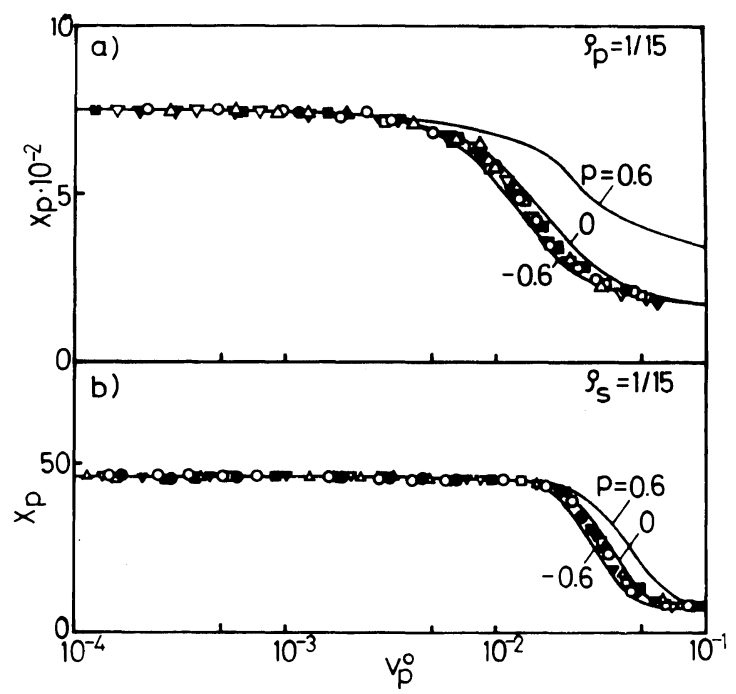

Figure 10. Dependence of $X_{\mathrm{p}}$ of the polymers in a polymer-rich phase a) or in a polymer-lean phase b) on the initial polymer volume fraction $v_{\mathrm{p}}{ }^{\circ}$. The original polymer, Schulz-Zimm distr., $X_{w}{ }^{\circ}=300, X_{w}{ }^{\circ} / X_{n}{ }^{\circ}=$ $2.0 ; \bigcirc, \chi_{12}=0.48, \chi_{13}=0.20, \chi_{23}=1.00 ; \bigcirc, \chi_{12}=0.48, \chi_{13}=0.20, \chi_{23}=1.2 ; \Delta, \chi_{12}=0.48, \chi_{13}=0.20, \chi_{23}=$ $0.80 ; \square, \chi_{12}=0.48, \chi_{13}=0.05, \chi_{23}=1.00 ; \square, \chi_{12}=0.48, \chi_{13}=0.50, \chi_{23}=1.00 ; \nabla, \chi_{12}=0.20, \chi_{13}=0.20$, $\chi_{23}=1.00 ; \nabla, \chi_{12}=1.30, \chi_{13}=0.20, \chi_{23}=1.00$. Full lines are for quasi-binary solutions and the concentration dependence of $\chi$-parameter, $p$ is denoted on the curve.

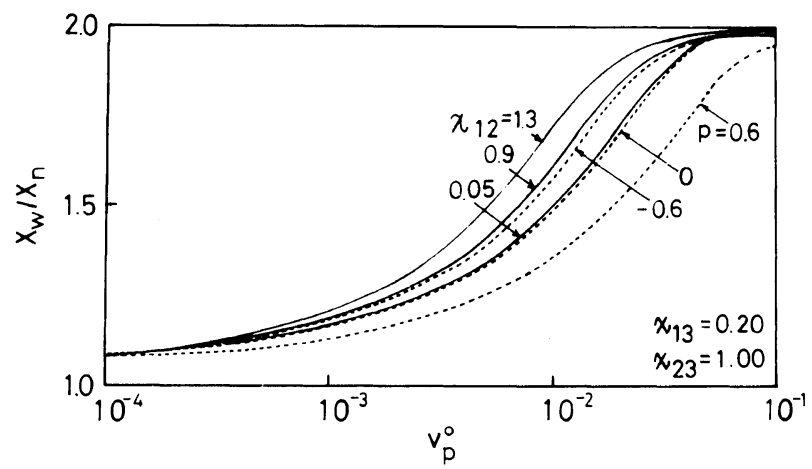

Figure 11. Effect of the initial polymer volume fraction $v_{\mathrm{p}}{ }^{\circ}$ on $X_{w} / X_{n}$ of the polymer in a polymer-rich phase. The original polymer, Schulz-Zimm distr., $X_{w}{ }^{\circ}=300, X_{w}{ }^{\circ} X_{n}{ }^{\circ}=2.0$; full line, quasi-ternary solution with $\chi_{13}=0.20, \chi_{23}=1.00 \chi_{12}$ denoted on curves; broken line, quasi-binary solution with the concentration dependence of $\chi$-parameter, $p$ is denoted on curves; the relative amount of polymer $\rho_{\mathrm{p}}=1 / 15$.

former, resulting in a decrease in the breadth of the MWD of the polymer with a decrease in $v_{\mathrm{p}}{ }^{\circ}$. Similar dependence of MWD on $v_{\mathrm{p}}{ }^{\circ}$ was observed for a multicomponent polymer-single solvent system.

In Figure 10, the $X_{\mathrm{p}}-v_{\mathrm{p}}{ }^{\circ}$ relations, obtained at constant $\rho_{\mathrm{p}}$ or $\rho_{\mathrm{s}}(1 / 15)$ for various combinations of $\chi_{12}, \chi_{13}$, and $\chi_{23}$ are shown.
Similar relations for quasi-binary solutions with various concentration dependence parameters $p$ in eq 5 are also shown as full lines for comparison. All the data for the quasi-ternary solutions yield a single master curve, irrespective of the combination of $\chi_{12}, \chi_{13}$, and $\chi_{23}$ values, lying between those for the quasibinary solutions with $p=0$ and -0.6 . These 


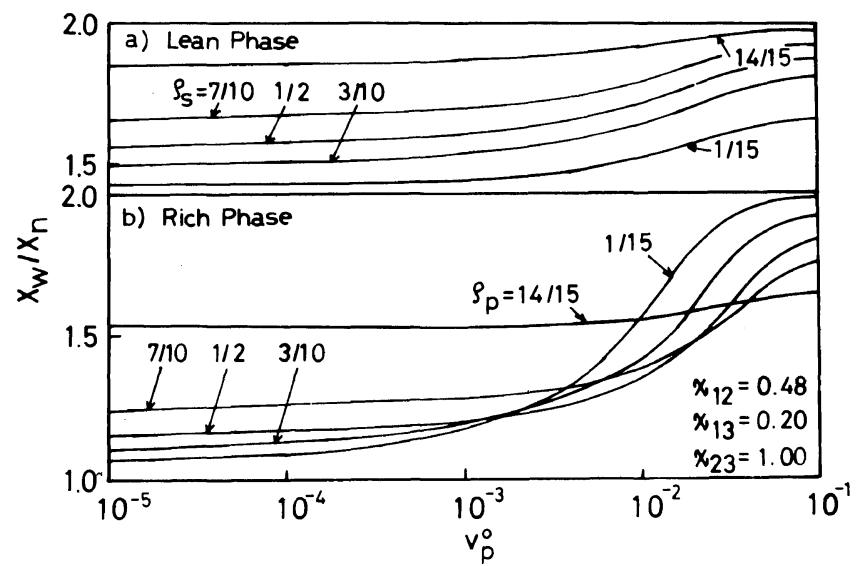

Figure 12. Effects of the relative amounts of polymers in a polymer-lean phase, $\rho_{\mathrm{s}}$, (number of curve in a)) and in a polymer-rich phase, $\rho_{\mathrm{p}}$, (number on curve in b)) on the relations between $X_{w} / X_{n}$ of the polymers and the initial polymer volume fraction. The original polymer, Schulz-Zimm distr., $X_{w}{ }^{\circ}=300$, $X_{w}{ }^{\circ} / X_{n}^{\circ}=2.0 ; \chi_{12}=0.48, \chi_{13}=0.20, \chi_{23}=1.00$.

plots demonstrate as asymptotic approach of $X_{\mathrm{p}}$ to a maximum value at lower $v_{\mathrm{p}}{ }^{\circ}$ and to a minimum at higher $v_{\mathrm{p}}{ }^{\circ} . X_{\mathrm{p}}$ can be regarded as roughly constant in the range $v_{\mathrm{p}}{ }^{\circ}<2 \times 10^{-3}$ for the polymer in the polymer-rich phase and $v_{\mathrm{p}}{ }^{\mathrm{o}}<2 \times 10^{-2}$ for the polymer in the polymerlean phase and $X_{\mathrm{p}}$ transits rapidly in a comparatively narrow $v_{\mathrm{p}}{ }^{\circ}$ range.

The value of $X_{w} / X_{n}$ of the polymer in the polymer-rich phase is plotted in Figure 11 vs. $\log v_{\mathrm{p}}{ }^{\circ}$. In this figure, the results of a quasibinary solution are shown as broken lines for comparison. Simply by a proper choice $\chi_{12}$, $\chi_{13}$, and $\chi_{23}$ values, polymers could not be prepared having narrower MWD than those obtained from a quasi-binary solution assuming $p=0$.

Unexpectedly, $X_{w} / X_{n}$ vs. $v_{\mathrm{p}}{ }^{\circ}$ relations for quasi-ternary solutions were found to be very similar in shape to those for quasi-binary solutions calculated with $p \leqq 0$. This suggests that a multicomponent polymer-solvent 1solvent 2 system can be approximated with a multicomponent polymers-solvent system (i.e., single solvent approximation) if the negative concentration dependence of $\chi$ parameter is assumed. For any system of polymer and solvent investigated, a negative $p$ has never been reported and hence is a hypothetical system.

Figure 12 shows the relation between $X_{w} / X_{n}$ of the polymer in the polymer-lean and -rich phases and $v_{\mathrm{p}}{ }^{\circ}$ for a given combination of three $\chi$ parameters when a constant $\rho_{\mathrm{p}}\left(\equiv 1-\rho_{\mathrm{s}}\right)$ of the polymers is separated. The MWD of the polymer in the polymer-lean phase becomes sharp without limit as $\rho_{\mathrm{s}}$ decreases and the effect of $\rho_{\mathrm{s}}$ predominates over $v_{\mathrm{p}}{ }^{\circ}$ on the polydispersity of the polymer-lean phase. As $\rho_{\mathrm{p}}$ decreases, the $v_{\mathrm{p}}{ }^{\circ}$ dependence of $X_{w} / X_{n}$ of the polymer in the polymer-rich phase becomes remarkable, as expected. The $\rho_{\mathrm{p}}$ value, at which the minimum $X_{w} / X_{n}$ is obtained at a given $v_{\mathrm{p}}^{\circ}, \rho_{\mathrm{p}, \min }$, depends on $v_{\mathrm{p}}^{\circ}$, becoming smaller as $v_{\mathrm{p}}{ }^{\circ}$ deminishes.

Figure 13 shows the normalized MWD curve of the polymers in the polymer-rich and -lean phases, $g_{(2)}(X)$ and $g_{(1)}(X)$, isolated from a quasi-ternary solution. With a decrease in $\rho_{\mathrm{p}}$, a remarkable increase in its content in a larger $X$ region and a significant lowering of the peak height are observed in both $g_{(2)}(X)$ and $g_{(1)}(X)$ and in the former, $X_{\mathrm{p}}$ shifts, noticably to a larger $X$ region, but in the latter remains constant, roughly identical with $X_{\mathrm{p}}$ of the original polymer. 


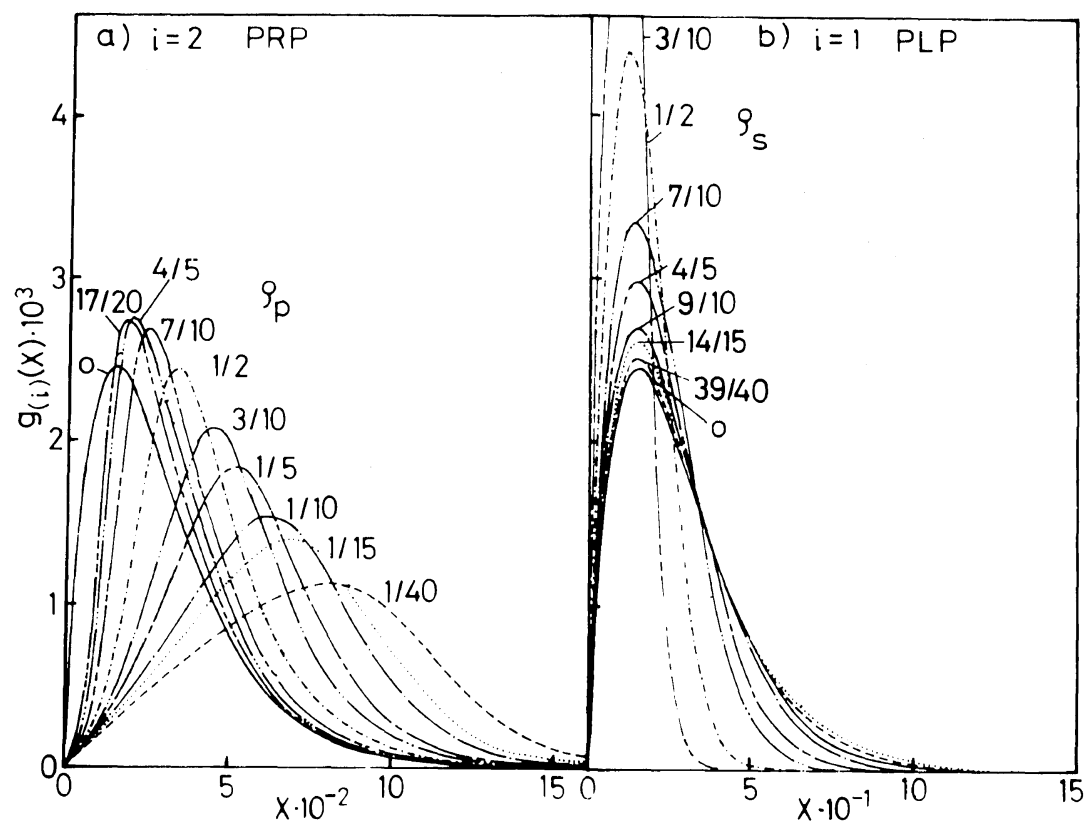

Figure 13. Effects of the relative amounts of polymers in a polymer-rich phase $\rho_{\mathrm{p}}$ (a) and in a polymerlean phase $\rho_{\mathrm{s}}$ (b) on the MWD curve $g_{(i)}(X)(i=1$ and 2) of the polymers. The original polymer, SchulzZimm distr., $X_{w}{ }^{\circ}=300, X_{w}{ }^{\circ} / X_{n}{ }^{\circ}=2.0$; the initial polymer volume fraction $v_{\mathrm{p}}{ }^{\circ}=0.005 ; \chi_{12}=0.48, \chi_{13}=0.20$, $\chi_{23}=1.00 ; \rho_{\mathrm{p}}$ and $\rho_{\mathrm{s}}$ are denoted on the curve.

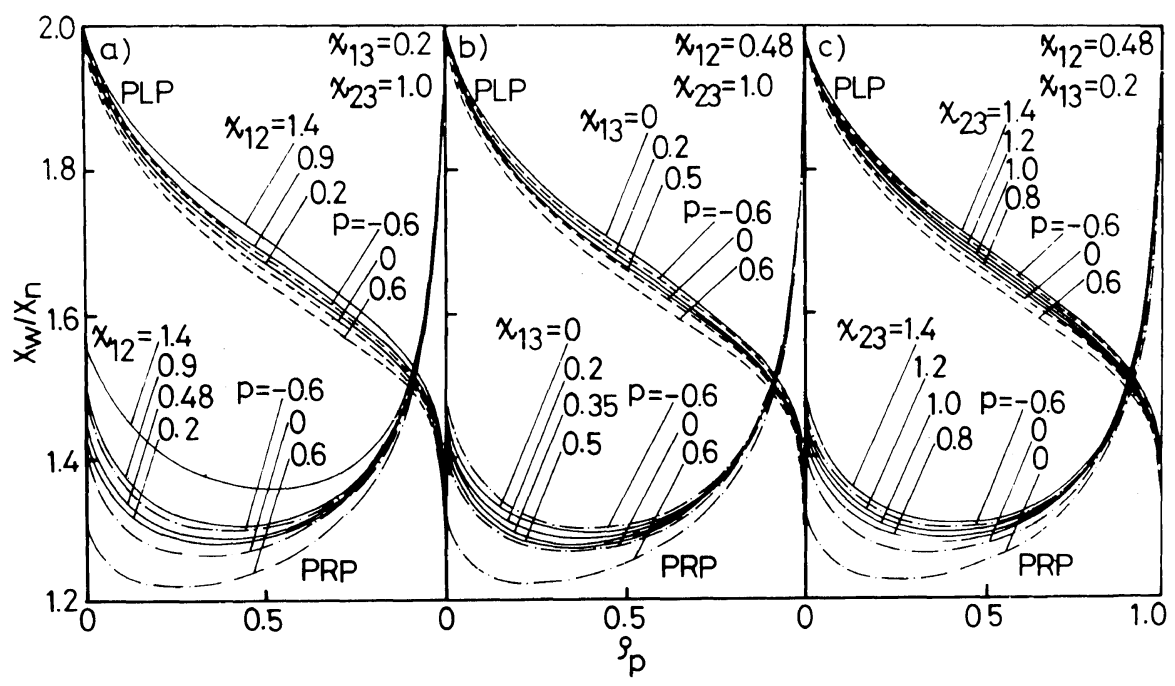

Figure 14. Effects of the parameters $\chi_{12}$ (a), $\chi_{13}$ (b) and $\chi_{23}$ (c) on the relations between $X_{w} / X_{n}$ of the polymers in a polymer-rich phase $\rho_{\mathrm{p}}$. The original polymer, Schulz-Zimm distr., $X_{w}{ }^{\circ}=300, X_{w}{ }^{\circ} / X_{n}{ }^{\circ}=2.0$; a) $\chi_{12}$ is denoted on curve, $\chi_{13}=0.2, \chi_{23}=1.0$; b) $\chi_{12}=0.48, \chi_{13}$ is denoted on curve, $\chi_{23}=1.0$; c) $\chi_{12}=0.48$, $\chi_{13}=0.20$ and $\chi_{23}$ is denoted on curve; broken line, quasi-binary solution, the concentration dependence of $\chi$-parameter $p$ is denoted on curve. 
Figure 14 shows the effects of $\chi_{12}, \chi_{13}$, and $\chi_{23}$ on the relationships between $X_{w} / X_{n}$ of the polymer dissolving in the polymer-rich phase and that remaining in the polymer-lean phase and $\rho_{\mathrm{p}}$ for $v_{\mathrm{p}}{ }^{\mathrm{o}}=0.005$. Figure 14 also shows the data for a quasi-binary system. $X_{w} / X_{n}$ of the polymer in the polymer-rich phase decreases first and then increases after passing through a minimum of $X_{w} / X_{n}\left(\left(X_{w} / X_{n}\right)_{\min }\right) . \rho_{\mathrm{p}}$, yielding $\left(X_{w} / X_{n}\right)_{\min }$, is defined as $\rho_{\mathrm{p}, \min }$. In contrast to this, $X_{w} / X_{n}$ of the polymer in the polymer-lean phase decreases monotonously with a decrease in $\rho_{\mathrm{s}}$, approaching 1.0 at the limit of $\rho_{\mathrm{s}}=0$ (i.e., $\left.\rho_{\mathrm{p}}=1\right)$. Figure 14 indicates that the characteristics observed in a quasi-binary systems hold even in a quasi-ternary (and probably much higher order) system. The $X_{w} / X_{n}$ of the polymers in both phases is without exception smaller in a quasi-binary system with $p>0$ than in quasi-ternary systems, suggesting that fractionation using quasi-binary system is much more effective compared to a quasiternary system.

Figure 15 shows the standard deviation $\sigma^{\prime}$ of the polymers in both phases as a function of $\rho_{\mathrm{p}}$ for quasi-ternary and -binary systems. In this case, for the former system, various combinations of $\chi_{12}, \chi_{13}$, and $\chi_{23}$ are assumed. For the same value of $\rho_{\mathrm{p}}$, the combination of these $\chi$ parameters controls to some extent the polydispersity of the polymers. A decrease in $\chi_{12}$ improves the separation efficiency. It should be noted that $\sigma^{\prime}$, a much more adequate parameter representing the polydispersity than the ratio $X_{w} / X_{n}$, is always larger in the polymer-rich phase than in the polymer-lean phase. This clearly indicates the superiority of the solution method against the precipitation method, even for quasi-ternary solutions as well as quasi-binary solutions.

Figure 16 illustrates the effect of $v_{\mathrm{p}}{ }^{\circ}$ on the polydispersity of the polymers in both phases. This effect is conspicuous for polymers remaining in the polymer-rich phase, especially at extremely small $\rho_{\mathrm{p}}$. A striking decrease in $\rho_{\mathrm{p}, \min }$ for both $X_{w} / X_{n}$ and $\sigma^{\prime}$ is noted with a

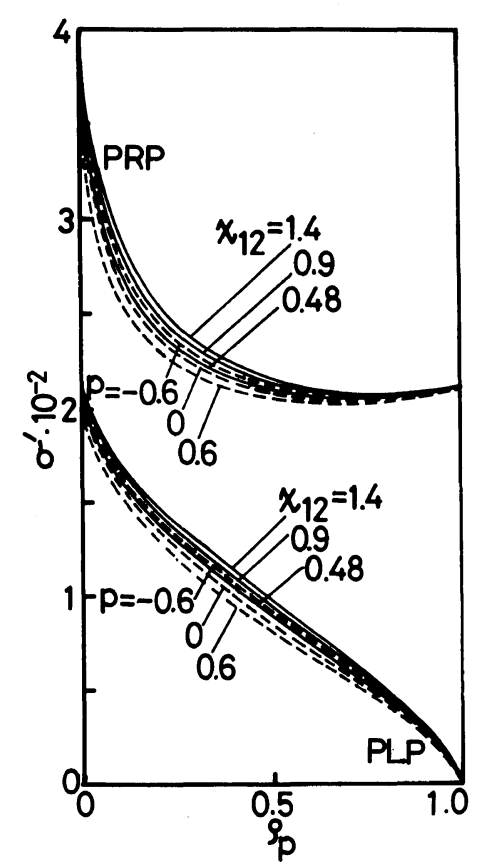

Figure 15. The standard deviation $\sigma^{\prime}$ of the polymers in a polymer-rich phase and in a polymer-lean phase separated from a quasi-ternary solution (full line) and quasi-binary solution (broken line), plotted $v s$. the relative amounts of polymers in a polymer-rich phase $\rho_{\mathrm{p}}$. The original polymer, Schulz-Zimm distr., $X_{w}{ }^{\circ}=300$, $X_{w}{ }^{\circ} / X_{n}{ }^{\circ}=2.0 ; \chi_{12}$, number on curve, $\chi_{13}=0.20, \chi_{23}=$ 1.00; initial polymer volume fraction $v_{\mathrm{p}}{ }^{\circ}=0.005$; for quasi-binary solution, the concentration dependence of $\chi$-parameter $p$, number on curve.

decrease in $v_{\mathrm{p}}{ }^{\circ}$.

Figure 17 shows the relationships between $\rho_{\mathrm{p}}$ and $v_{\mathrm{p}}{ }^{\mathrm{o}}$, which yield the polymers, in the polymer-rich and -lean phases, having the same value of $X_{w} / X_{n}$. As expected from Figure 14 and 16, two different values of $\rho_{\mathrm{p}}$ at a given value of $v_{\mathrm{p}}{ }^{\circ}$ yield the same $X_{w} / X_{n}$ of the polymer-rich phase. In the range of small $\rho_{\mathrm{p}}$ (strictly speaking, in a region lower than the broken line), $X_{w(2)} / X_{n(2)}$ of the polymer depends mainly on $v_{\mathrm{p}}^{\circ}$, while in the higher $\rho_{\mathrm{p}}$ range (i.e., a region higher than the broken line), $X_{w(2)} / X_{n(2)}$ is predominantly governed by $\rho_{\mathrm{p}}$ alone. $X_{w(1)} / X_{n(1)}$ is $\rho_{\mathrm{p}^{-}}$(i.e., $\left.\rho_{\mathrm{s}^{-}}\right)$dependent over the whole range of $v_{\mathrm{p}}{ }^{\circ}$. Figure 17 offers the fundamental information necessary for 

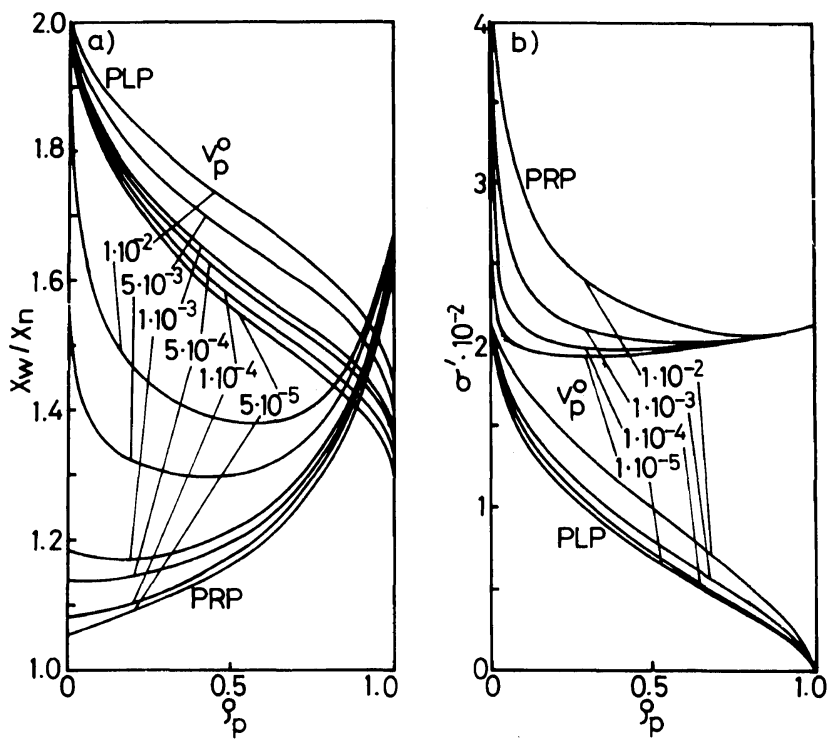

Figure 16. Effect of the initial polymer volume fraction $v_{\mathrm{p}}{ }^{\circ}$ (number on curve) on relations between $X_{w} / X_{n}$ or $\sigma^{\prime}$ of the polymers in a polymer-rich and -lean phases and $\rho_{\mathrm{p}}$ for quasi-ternary solution. The original polymer, Schulz-Zimm distr., $X_{w}{ }^{\circ}=300, X_{w}{ }^{\circ} / X_{n}{ }^{\circ}=2.0 ; \chi_{12}=0.48, \chi_{13}=0.20, \chi_{23}=1.30$.

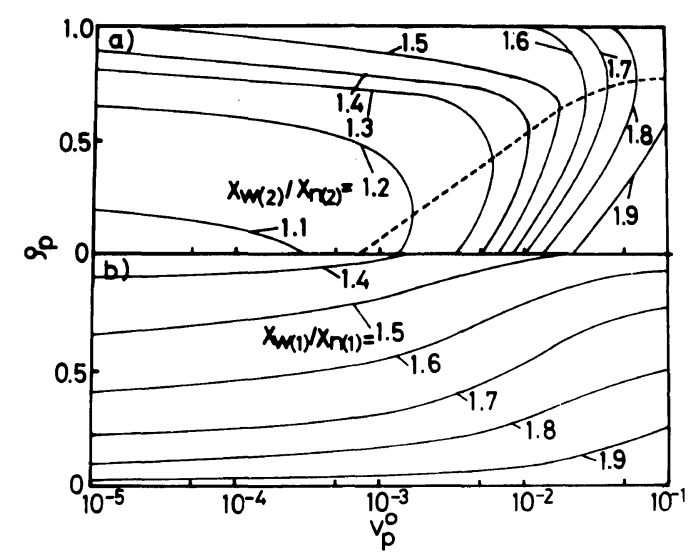

Figure 17. Relations between initial polymer volume fraction $v_{\mathbf{p}}{ }^{\mathrm{o}}$, the relative amount of the polymer-rich phase $\rho_{\mathrm{p}}$ and $X_{w} / X_{n}$ of the polymer in a polymer-rich phase (upper part) and in a polymer-lean phase (lower part), both separated from quasi-ternary solution. The original polymer, Schulz-Zimm distr., $X_{w}{ }^{\circ}=300$, $X_{w}{ }^{\circ} / X_{n}{ }^{\circ}=2.0 ; \chi_{12}=0.48, \chi_{13}=0.20, \chi_{23}=1.30$.

designing a detailed fractionation scheme for a given polymer and a given combination of solvent 1 and solvent 2 .

Figure 18 shows the $v_{\mathrm{p}}{ }^{\circ}$ dependence of the volume fractions of solvent 1 in the polymerlean phase and in the polymer-rich phase, $v_{1(1)}$ and $v_{1(2)}$, and the volume fraction of solvent 2 in the two phases, $v_{2(1)}$ and $v_{2(2)}$ for a given combination of $\chi_{12}, \chi_{13}$, and $\chi_{23} . v_{1(1)}, v_{1(2)}$, and $v_{2(2)}$ increase, but $v_{2(1)}$ decreases with an increase in $v_{\mathrm{p}}{ }^{\mathrm{o}}$. The volume fractions of solvents in the two phases are markedly influenced by the $\chi_{23}$ parameter. An increase in $\chi_{23}$ (i.e., the precipitation power) brings about a significant increase in $v_{1(1)}$ and $v_{1(2)}$ and a decrease in $v_{2(1)}$ and $v_{2(2)}$.

Figure 19 shows the relations between the polymer volume fraction, $v_{\mathrm{p}(\mathbf{1})}$, in the polymerlean phase, separated from quasi-ternary polymer solutions having various combinations of $\chi_{12}, \chi_{13}$, and $\chi_{23}$ at various $\rho_{\mathrm{p}} . v_{\mathrm{p}(1)}$ is overwhelmingly dependent on $\rho_{\mathrm{p}}$ and $v_{\mathrm{p}}{ }^{\circ}$ and substantially independent of the three $\chi$ parameters. The full lines in Figure 19 are the results of a quasi-binary solution, with $p=$ $-0.6 \sim+0.6$ on each line. These lines are completely consistent with those of a quasiternary solution. Large variation in $\mathrm{p}$ for $\mathrm{a}$ 


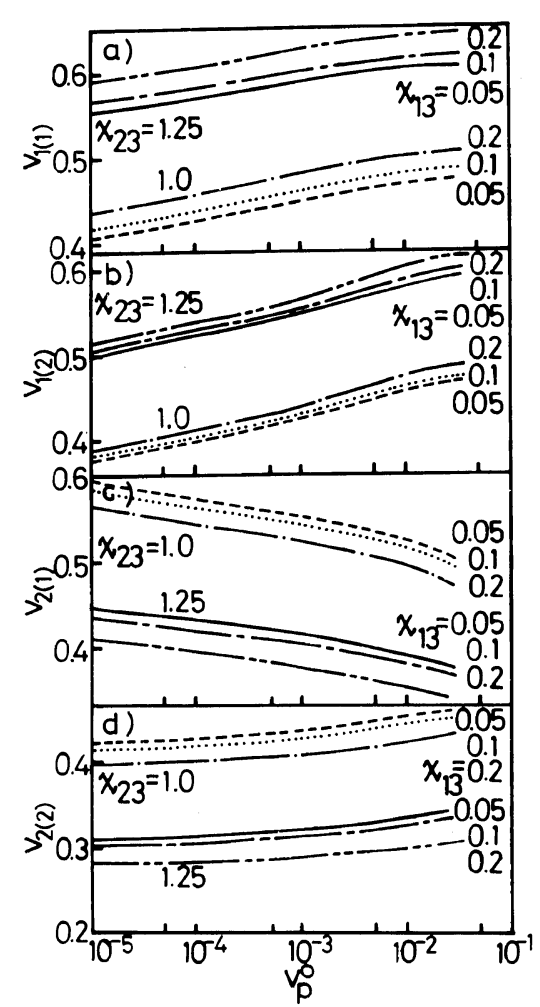

Figure 18. The volume fractions of solvent 1 in polymer-lean and -rich phases $v_{1(1)}$ a) and $v_{1(2)}$ b), and the volume fractions of solvent 2 in polymer-lean and -rich phases $v_{2(1)} \mathrm{c}$ ) and $v_{2(2)} \mathrm{d}$ ) plotted against the initial polymer volume fraction $v_{\mathrm{p}}{ }^{\circ}$. The original polymer, Schulz-Zimm distr., $X_{w}{ }^{\circ}=300, X_{w}{ }^{\circ} / X_{n}{ }^{\circ}=2.0$; the relative amount of the polymer in a polymer-rich phase, $\rho_{\mathrm{p}}=1 / 15 ;-, \chi_{12}=0.48, \chi_{13}=0.05, \chi_{23}=1.25 ;--\cdots$, $\chi_{12}=0.48, \chi_{13}=0.10, \chi_{23}=1.25 ;-\cdots-, \chi_{12}=0.48, \chi_{13}=$ $0.20, \chi_{23}=1.25 ;-\cdots-\cdots, \chi_{12}=0.48, \chi_{13}=0.05, \chi_{23}=1.00$; $\cdots \cdots \cdots, \chi_{12}=0.48, \chi_{13}=0.10, \chi_{23}=1.00 ;-\cdot-, \chi_{12}=0.48$, $\chi_{13}=0.20, \chi_{23}=1.00$.

quasi-binary solution at given $\rho_{\mathrm{p}}$ does not give a significant change in the $v_{\mathrm{p}(1)}-v_{\mathrm{p}}{ }^{0}$ relations.

Figure 20 and 21 depict the relations among the polymer volume fraction, $v_{\mathrm{p}(2)}$, in the polymer-rich phase and $v_{\mathrm{p}}{ }^{\circ}$ and $\rho_{\mathrm{p}}$. Under the same operating conditions, such as $\chi_{12}, \chi_{13}$, and $\chi_{23}$, and $\rho_{\mathrm{p}}(=1 / 15)\left(\right.$ or $\left.v_{\mathrm{p}}{ }^{\mathrm{o}}=0.005\right), v_{\mathrm{p}(2)}$ decreases with an increase in $v_{\mathrm{p}}{ }^{\circ}$ and with a decrease in $\rho_{\mathrm{p}}$. Unlike $v_{\mathrm{p}(1)}, v_{\mathrm{p}(2)}$ is evidently dependent on the $\chi$ parameters, and $v_{\mathrm{p}(2)}$, and accordingly, $v_{\mathrm{p}(2)}-v_{\mathrm{p}(1)}$, are larger for smaller

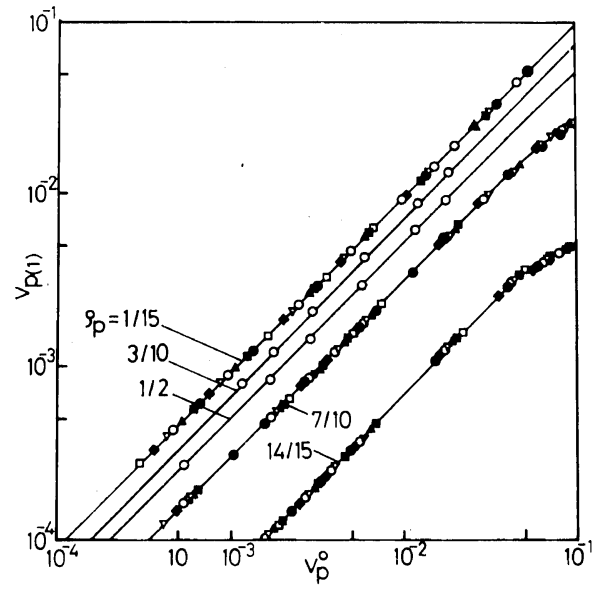

Figure 19. Plot of the polymer volume fraction of the polymer in a polymer-lean phase $v_{\mathrm{p}(1)}$ against the initial polymer volume fraction $v_{\mathrm{p}}{ }^{\circ}$ at a constant relative amount of the polymer partitioned in a polymer-rich phase $\rho_{\mathrm{p}}$ (number on curve). The original polymer, Schulz-Zimm distr., $X_{w}{ }^{\circ}=300, X_{w}{ }^{\circ} / X_{n}{ }^{\circ}=2.0 ; 0, \chi_{12}=$ $0.48, \chi_{13}=0.20, \chi_{23}=0.80 ; \bigcirc, \chi_{12}=0.48, \chi_{13}=0.20$, $\chi_{23}=1.00 ; \square, \chi_{12}=0.48, \chi_{13}=0.20, \chi_{23}=1.20 ; \nabla, \chi_{12}=$ $0.48, \chi_{13}=0.05, \chi_{23}=1.0 ; \square, \chi_{12}=0.48, \chi_{13}=0.50, \chi_{23}=$ $1.00 ; \boldsymbol{\Delta}, \chi_{12}=0.20, \chi_{13}=0.20, \chi_{23}=1.00 ; \diamond, \chi_{12}=1.30$, $\chi_{13}=0.20, \chi_{23}=1.00$.

$\chi_{12}$, larger $\chi_{13}$ and smaller $\chi_{23}$. The quantity $v_{\mathrm{p}(2)}-v_{\mathrm{p}(1)}$ is a measure of the ease of separation of the polymers. ${ }^{9}$ The dotted lines in Figure 20 result from a quasi-binary solution. $v_{\mathrm{p}(2)}$ of the quasi-ternary solution can never exceed that of the quasi-binary solution with $p=0$, suggesting an effective separation of the two phases for quasi-ternary system to be comparatively difficult compared to that for quasi-binary system.

It is intuitively expected that solvent 1, which has a large affinity to the polymer, is more or less selectively partitioned into a polymer-rich phase, that is, a so-called selective adsorption will occur in the phase equilibrium of the multicomponent polymer-solvent 1-solvent 2 system.

Here, we define $\theta_{2}$ as a measure representing the selective adsorption by eq 6

$$
\theta_{2}=\frac{v_{2(1)} /\left(v_{1(1)}+v_{2(1)}\right)}{v_{2(2)} /\left(v_{1(2)}+v_{2(2)}\right)}
$$




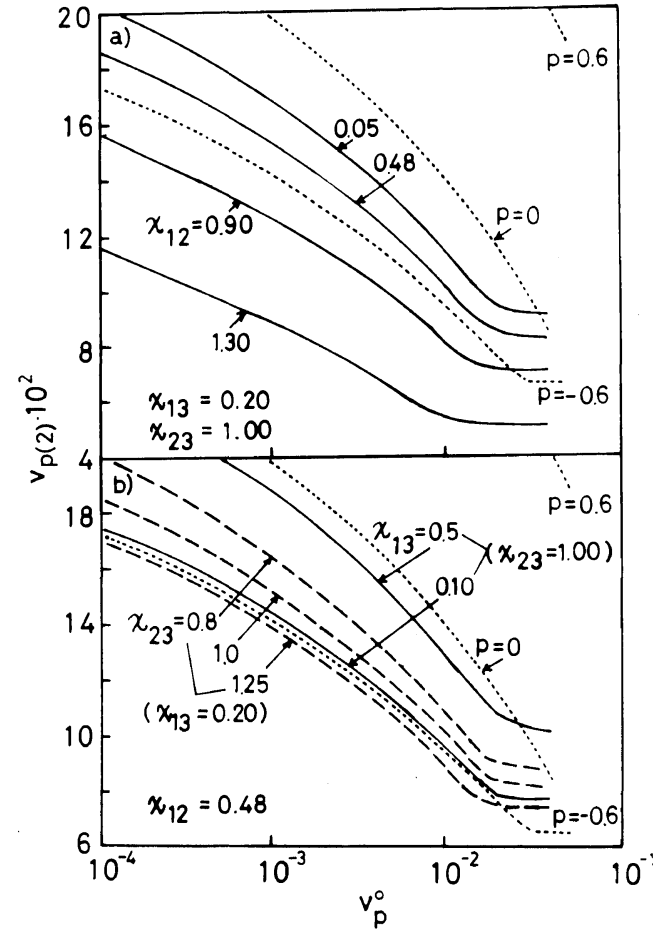

Figure 20. Plot of the polymer volume fraction in a polymer-rich phase $v_{\mathrm{p}(2)}$ versus the initial polymer volume fraction $v_{\mathrm{p}}{ }^{\circ}$ for quasi-ternary (full and broken lines) and quasi-binary (dotted line) solutions at constant relative amount of the polymer $\rho_{\mathrm{p}}$. The original polymer, Schulz-Zimm distr., $X_{w}{ }^{\circ}=300, X_{w}{ }^{\circ} / X_{n}{ }^{\circ}=2.0$; for a quasi-ternary solution, a) $\chi_{12}$, numbers on curve, $\chi_{13}=$ $\left.0.20, \chi_{23}=1.00, b\right)$ full line, $\chi_{12}=0.48, \chi_{13}$, numbers on the curve, $\chi_{23}=1.00$, broken line, $\chi_{12}=0.48, \chi_{13}=0.20$, $\chi_{23}$, numbers on the curve; for a quasi-binary solution, the concentration dependence of $\chi$-parameter, $p$ is denoted on curve.

Figure 22 demonstrates the dependence of $\theta_{2}$ on $v_{\mathrm{p}}{ }^{\mathrm{o}}$. It is of interest to note that the selective adsorption becomes remarkable for larger $\chi_{12}$, smaller $\chi_{13}$ and larger $\chi_{23}$ (i.e., when very good solvent and very poor solvent are employed), especially in a lower $v_{\mathrm{p}}{ }^{\mathrm{o}}$ region. The selective adsorption becomes negligible in relatively concentrated solution.

Figure 23 shows the relations between $\theta_{2}$ and $\rho_{\mathrm{p}}$ for the quasi-ternary solutions of $v_{\mathrm{p}}{ }^{\circ}=$ 0.005 . The magnitude of $\theta_{2}$ increases with an increase in $\rho_{\mathrm{p}}$. The slope of the plots, $\mathrm{d} \theta_{2} / \mathrm{d} \rho_{\mathrm{p}}$ is

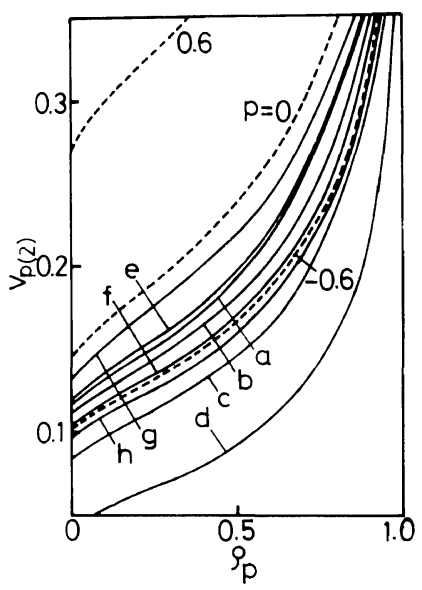

Figure 21. The polymer volume fraction in a polymerrich phase $v_{\mathrm{p}(2)}$ plotted as a function of the relative amount of the polymer in a polymer-rich phase $\rho_{\mathrm{p}}$. The original polymer, Schulz-Zimm distr., $X_{w}{ }^{\circ}=300$, $X_{w}{ }^{\circ} / X_{n}{ }^{\circ}=2.0$, initial polymer volume fraction $v_{\mathrm{p}}{ }^{\circ}=$ 0.005 ; full line, quasi-ternary solution; $a, \chi_{12}=0, \chi_{13}=$ $0.20, \chi_{23}=1.0 ; \mathrm{b}, \chi_{12}=0.48, \chi_{13}=0.20, \chi_{23}=1.0 ; \mathrm{c}, \chi_{12}=$ $0.90, \chi_{13}=0.20, \chi_{23}=1.0 ; \mathrm{d}, \chi_{12}=1.4, \chi_{13}=0.2, \chi_{23}=1.0$; e, $\chi_{12}=0.48, \chi_{13}=0.20, \chi_{23}=0.80 ; \mathrm{f}, \chi_{12}=0.48, \chi_{13}=$ $0.20, \chi_{23}=1.30 ; \mathrm{g}, \chi_{12}=0.48, \chi_{13}=0.50, \chi_{23}=1.00 ; \mathrm{h}$, $\chi_{12}=0.48, \chi_{13}=0, \chi_{23}=1.0$.

nearly constant at constant $\rho_{\mathrm{p}}$ over a wide range of three $\chi$ parameters. Inspection of Figures 22 and 23 leads to the conclusion that $\chi_{23}$ plays the most important role in selective adsorption.

In this study, it has been shown that parameters characterizing the phase separation of the quasi-ternary solutions, such as $\sigma, R$, $v_{\mathrm{p}(1)}, v_{\mathrm{p}(2)}, X_{w(1)} / X_{n(1)}, X_{w(2)} / X_{n(2)}, \sigma_{(1)}^{\prime}$ and $\sigma_{(2)}^{\prime}$ can be equated to those for quasi-binary solutions by assuming an adequate concentration dependence $p$ of the $\chi$ parameter. Then, for various equilibrium parameters, a nearly common $p$ value can be reasonably chosen if the three $\chi_{\mathrm{ij}}$ parameters are given in advance. For example, for a quasi-ternary system with $\chi_{12}=$ $0.2, \chi_{13}=0.2$, and $\chi_{23}=1.0, p=-0.26 \pm 0.02$ was estimated, when the polymer with the Schulz-Zimm distribution $\left(X_{w}{ }^{\circ}=300\right)$ and dissolved in a solution of $v_{\mathrm{p}}{ }^{\mathrm{o}}=0.005$ was separated into two phases. 


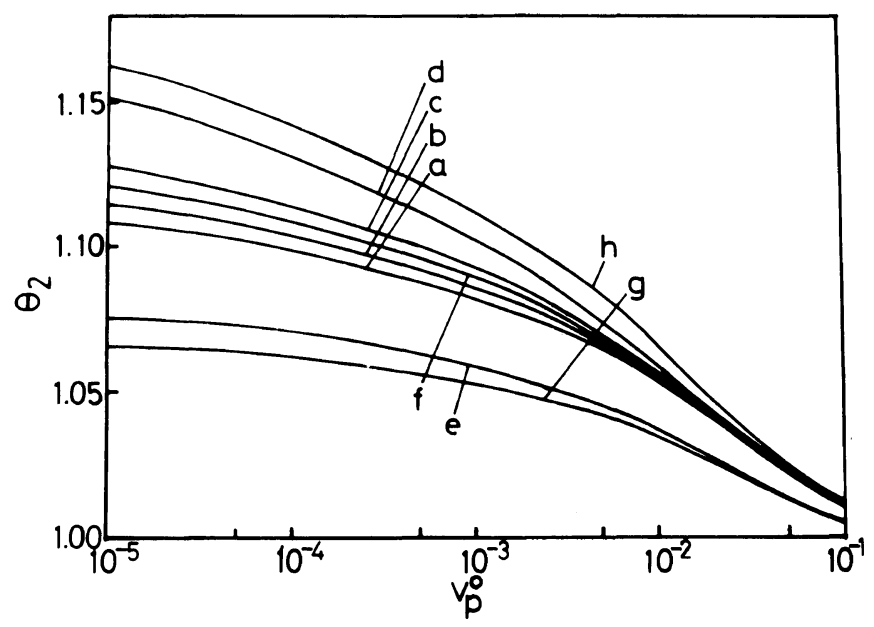

Figure 22. Dependence of the selective adsorption $\theta_{2}$ on the initial polymer volume fraction $v_{\mathrm{p}}{ }^{\circ}$ for a quasi-ternary system. The original polymer, Schulz-Zimm distr., $X_{w}{ }^{\circ}=300, X_{w}{ }^{\circ} / X_{n}{ }^{\circ}=2.0$; the relative amount of the polymer in a polymer-rich phase, $\rho_{\mathrm{p}}=1 / 15$; $\mathrm{a}, \chi_{12}=0.2, \chi_{13}=0.2, \chi_{23}=1.00 ; \mathrm{b}, \chi_{12}=0.48$, $\chi_{13}=0.20, \chi_{23}=1.00 ; \mathrm{c}, \chi_{12}=0.90, \chi_{13}=0.20, \chi_{23}=1.00 ; \mathrm{d}, \chi_{12}=1.30, \chi_{13}=0.2, \chi_{23}=1.00 ; \mathrm{e}, \chi_{12}=0.48$, $\chi_{13}=0.50, \chi_{23}=1.00 ; \mathrm{f}, \chi_{12}=0.48, \chi_{13}=0.05, \chi_{23}=1.00 ; \mathrm{g}, \chi_{12}=0.48, \chi_{13}=0.20, \chi_{23}=0.80 ; \mathrm{h}, \chi_{12}=0.48$, $\chi_{13}=0.20, \chi_{23}=1.20$.

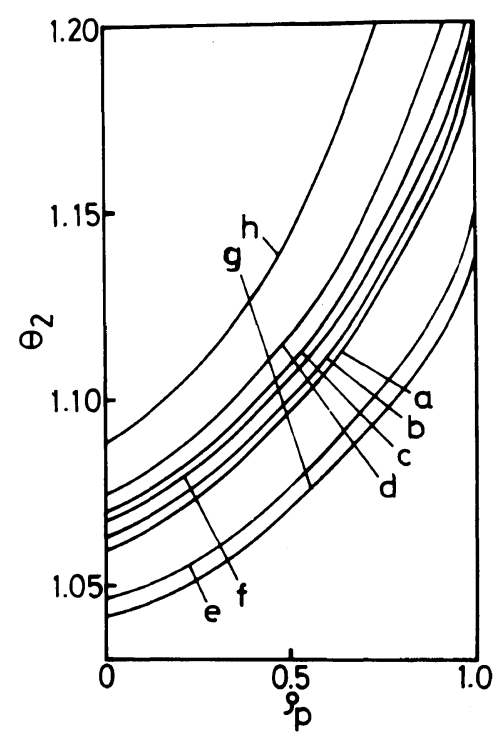

Figure 23. Dependence of the selective adsorption $\theta_{2}$ on the relative amount of the polymer in a polymer-rich phase $\rho_{\mathrm{p}}$ for quasi-ternary solutions with $v_{\mathrm{p}}{ }^{\mathrm{o}}=0.005$. The original polymer Schulz-Zimm distr., $X_{w}{ }^{0}=300$, $X_{w}{ }^{\circ} / X_{n}{ }^{\circ}=2.0$. Key to lines $\mathrm{a}-\mathrm{h}$ is given in Figure 22 .

Figure 24 shows the relations among $\chi_{12}$, $\chi_{13}$, and $\chi_{23}$, yielding the same phase separation characteristics as those obtained for the

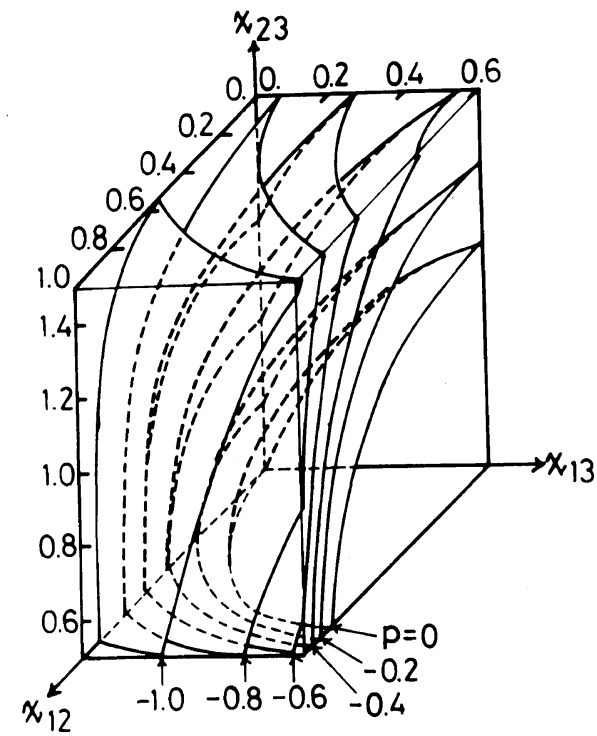

Figure 24. Relationships between $\chi_{12}, \chi_{13}$, and $\chi_{23}$, yielding the same phase separation characteristics as those by a quasi-binary solution with the concentration dependence of the $\chi$-parameter $\mathrm{p}$ (numbers on the curve). The original polymer, Schulz-Zimm distr., $X_{w}{ }^{\circ}=$ $300, X_{w}{ }^{\circ} / X_{n}{ }^{\circ}=2.0$; the initial polymer volume fraction $v_{\mathrm{p}}{ }^{\mathrm{o}}=0.005$; relative amount of the polymer-rich phase $\rho_{\mathrm{p}}=1 / 15$. 


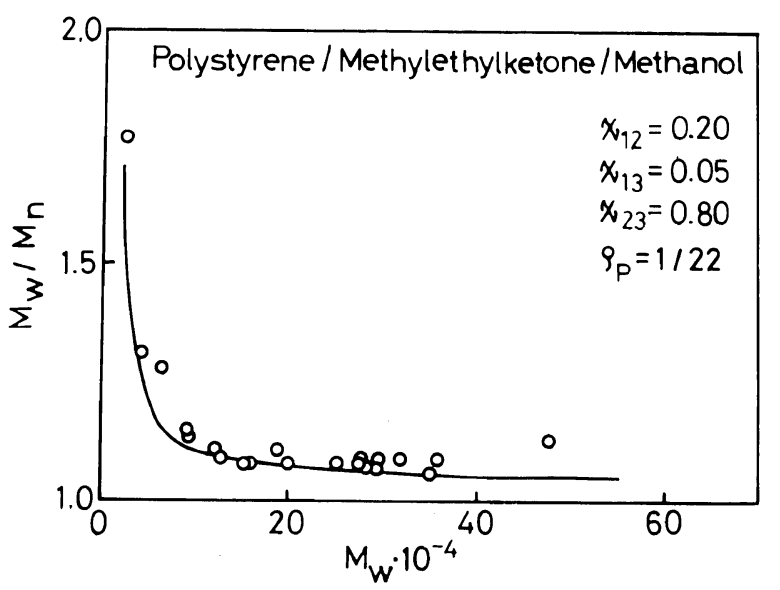

Figure 25. The ratio $M_{w} / M_{n}$ plotted against $M_{w}$ of the fractions separated by successive solution fractionation from a quasi-ternary system. $\bigcirc$, experimental data on polystyrene-methylethylketonemethanol; full line, theoretical curve $\left(\chi_{12}=0.20, \chi_{13}=0.05, \chi_{23}=0.80\right.$ and $\left.\rho_{\mathrm{p}}=1 / 22\right) ; v_{\mathrm{p}}{ }^{0}=6.15 \cdot 10^{-3}$.

quasi-binary solutions with fixed $p$ value. The quasi-ternary solutions having small $\chi_{12}$, large $\chi_{13}$, and large $\chi_{23}$ can be reasonably approximated with a quasi-binary solution with large negative $p$. A combination of small $\chi_{12}$ and large $\chi_{13}$ or that of large $\chi_{12}$ and small $\chi_{13}$ corresponds to a fixed $p$, if $\chi_{23}$ is maintained constant. This relation between $\chi_{12}$ and $\chi_{13}$ belongs to a family of hyperbolas. A suitable relation between $\chi_{13}$ and $\chi_{23}$, corresponding to a quasi-binary solution with fixed $p$, is almost $\chi_{13}$-dependent, especially for small $p$. A large $\chi_{23}$ is combined with large $\chi_{12}$ at constant $\chi_{13}$ if the quasi-ternary solution is approximated with the quasi-binary solution with fixed $p$. Conclusively, a quasi-ternary polymer solution gives smaller $\sigma$, smaller $R$, and a broader molecular weight distribution of the polymers partitioned in the two phase, compared to a quasi-binary polymer solution in which the polymer having the same molecular characteristics as that used in a quasi-ternary solution is dissolved. This strongly suggests that the fundamental characteristics observed for the twophase equilibrium of a quasi-binary polymer solution can be generalized quantitatively to a quasi-ternary polymer solution by assuming negative $p$, which is unrealistic for a quasi- binary polymer solution. A quasi-ternary polymer solution is inferior in separation efficiency, when the solution is used for fractionation.

Figure 25 shows the plots of $M_{w} / M_{n} v s . M_{w}$ for successive solution fractionation runs. The unfilled circles are a series of polystyrene fractions (total number $n=22$ ), separated successively using methylethylketone as solvent and methanol as non-solvent $\left(v_{\mathrm{p}}{ }^{\mathrm{o}}=6.15 \times\right.$ $\left.10^{-3}\right) .{ }^{16}$ The full line is a theoretical curve calculated assuming $\chi_{12}=0.2, \chi_{13}=0.05, \chi_{23}=$ 0.80 , and $\rho_{\mathrm{p}} \cong 1 / n(=1 / 22)$. The coincidence of the experimental data with theoretical calculation is satisfactory. However, note that the three $\chi_{i j}$ parameters are not experimentally determined and this figure does not mean a direct comparison between experimental evidence and the theory of phase separation of quasi-ternary solutions. This problem is still open for further research.

Acknowledgement. The authors should like to express their sincere gratitude to Dr. Yukio Miyazaki of Textile Research Laboratory, Asahi Chemical Industry Company, Ltd. for his helpful comments. 


\section{REFERENCES}

1. K. Kamide, S. Matsuda, and Y. Miyazaki, Polym. J., 16, 479 (1984).

2. K. Kamide and K. Sugamiya, Makromol. Chem., 139, 197 (1970).

3. K. Kamide, Y. Miyazaki, and K. Sugamiya, Makromol. Chem., 173, 133 (1973).

4. K. Kamide, Y. Miyazaki, and K. Yamaguchi, Makromol. Chem., 173, 157 (1973).

5. K. Kamide, Y. Miyazaki, and K. Yamaguchi, Makromol. Chem., 173, 175 (1973).

6. K. Kamide and Y. Miyazaki, Makromol. Chem., 176, 1029 (1975).

7. K. Kamide and Y. Miyazaki, Makromol. Chem., 176, 1051 (1975).

8. K. Kamide and Y. Miyazaki, Makromol. Chem., 176,
1427 (1975).

9. K. Kamide and Y. Miyazaki, Makromol. Chem., 176, 1447 (1975).

10. K. Kamide and Y. Miyazaki, Makromol. Chem., 176, 3453 (1975).

11. K. Kamide and Y. Miyazaki, Polym. J., 13, 325 (1981).

12. K. Kamide, T. Abe, and Y. Miyazaki, Polym. J., 14, 355 (1982).

13. J. N. Bronsted and K. Volqvartz, Trans. Faraday Soc., 36, 619 (1940).

14. G. V. Schulz and B. Jirgensons, Z. Phys. Chem., B46, 105 (1940).

15. K. Kamide and K. Sugamiya, Makromol. Chem., 156, 259 (1972).

16. K. Kamide, Y. Miyazaki, and T. Abe, Br. Polym. J., 13, 168 (1981). 